\title{
A SHARP SLOPE INEQUALITY FOR GENERAL STABLE FIBRATIONS OF CURVES
}

\author{
ATSUSHI MORIWAKI
}

\begin{abstract}
Let $\overline{\mathcal{M}}_{g}$ be the moduli space of stable curves of genus $g \geq 2$. Let $\Delta_{i}$ be the irreducible component of the boundary of $\overline{\mathcal{M}}_{g}$ such that general points of $\Delta_{i}$ correspond to stable curves with one node of type $i$. Let $\overline{\mathcal{M}}_{g}^{0}$ be the set of stable curves that have at most one node of type $i>0$. Let $\delta_{i}$ be the class of $\Delta_{i}$ in $\operatorname{Pic}\left(\overline{\mathcal{M}}_{g}\right) \otimes \mathbb{Q}$ and $\lambda$ the Hodge class on $\overline{\mathcal{M}}_{g}$. In this paper, we will prove a sharp slope inequality for general stable fibrations. Namely, if $C$ is a complete curve on $\overline{\mathcal{M}}_{g}^{0}$, then $\left((8 g+4) \lambda-g \delta_{0}-\sum_{i=1}^{[g / 2]} 4 i(g-i) \delta_{i} \cdot C\right) \geq 0$. As an application, we can prove effective Bogomolov's conjecture for general stable fibrations.
\end{abstract}

\section{Contents}

$\begin{array}{ll}1 . & \text { Introduction }\end{array}$

2. Preliminaries 3

2.1. Canonical linear systems of reduced curves with only node singularities $\quad 3$

\begin{tabular}{lll}
\hline 2.2. & Bogomolov inequality for fiber spaces & 6
\end{tabular}

3. Proof of Theorem 1.1 6

4. Calculation of invariants arising from Green functions 12

\begin{tabular}{lll}
\hline $5 . \quad$ Bogomolov conjecture over function fields & 16
\end{tabular}

\begin{tabular}{ll}
\hline References & 18
\end{tabular}

\section{INTRODUCTION}

Let $k$ be an algebraically closed field. Throughout this paper, we will fix this field $k$.

Let $X$ be a smooth projective surface over $k, Y$ a smooth projective curve over $k$, and $f: X \rightarrow Y$ a generically smooth semistable curve over $Y$ of genus $g \geq 2$. Let $P$ be a node of a singular fiber $X_{y}$ over $y$. We can assign a number $i$ to the node $P$ in the following way. Let $\iota: X_{y}^{\prime} \rightarrow X_{y}$ be the partial normalization of $X_{y}$ at $P$. If $X_{y}^{\prime}$ is connected, then $i=0$. Otherwise, $i$ is the minimum of arithmetic genera of two connected components of $X_{y}^{\prime}$. We say the node $P$ of the singular fiber $X_{y}$ is of type $i$. We denote by $\delta_{i}$ the number of nodes of type $i$ in singular fibers. If $\operatorname{char}(k)=0$, we know the following inequality due to Cornalba-Harris [2] and Xiao [1]]:

$$
(8 g+4) \operatorname{deg}\left(f_{*}\left(\omega_{X / Y}\right)\right) \geq g \delta
$$

where $\delta=\delta_{0}+\delta_{1}+\cdots+\delta_{[g / 2]}$. (By virtue of [8], this holds even if $\operatorname{char}(k)>0$.) This inequality is actually sharp because we know an example which attains equality of the above

Date: 1995, December (Version 1.0). 
inequality. When we consider a fibration with reducible fibers, we can however observe that $(8 g+4) \operatorname{deg}\left(f_{*}\left(\omega_{X / Y}\right)\right)$ is rather larger than $g \delta$. According to the exact formula [2, Proposition 4.7] for hyperelliptic fibrations, we can guess a sharper inequality:

$$
(8 g+4) \operatorname{deg}\left(f_{*}\left(\omega_{X / Y}\right)\right) \geq g \delta_{0}+\sum_{i=1}^{\left[\frac{g}{2}\right]} 4 i(g-i) \delta_{i} .
$$

In this paper, we would like to prove this sharper inequality for general stable fibrations.

Theorem $1.1(\operatorname{char}(k)=0)$. Let $\bar{f}: \bar{X} \rightarrow Y$ be the stable model of $f: X \rightarrow Y$. If every singular fiber of $\bar{f}: \bar{X} \rightarrow Y$ has at most one node of type $i>0$, then

$$
(8 g+4) \operatorname{deg}\left(f_{*}\left(\omega_{X / Y}\right)\right) \geq g \delta_{0}+\sum_{i=1}^{\left[\frac{g}{2}\right]} 4 i(g-i) \delta_{i} .
$$

In other words, we have the following. Let $\overline{\mathcal{M}}_{g}$ be the moduli space of stable curves of genus $g \geq 2$ over $k$. Let $\Delta_{i}$ be the irreducible component of the boundary of $\overline{\mathcal{M}}_{g}$ such that general points of $\Delta_{i}$ correspond to stable curves with one node of type $i$. Let $\overline{\mathcal{M}}_{g}^{0}$ be the set of stable curves that have at most one node of type $i>0$. Let $\delta_{i}$ be the class of $\Delta_{i}$ in $\operatorname{Pic}\left(\overline{\mathcal{M}}_{g}\right) \otimes \mathbb{Q}$ and $\lambda$ the Hodge class on $\overline{\mathcal{M}}_{g}$. Then, for all complete curves $C$ on $\overline{\mathcal{M}}_{g}^{0}$,

$$
\left((8 g+4) \lambda-g \delta_{0}-\sum_{i=1}^{\left[\frac{g}{2}\right]} 4 i(g-i) \delta_{i} \cdot C\right) \geq 0 .
$$

An idea of the proof of Theorem 1.1 is as follow. Let $E$ be the kernel of the natural homomorphism $f^{*} f_{*}\left(\omega_{X / Y}\right) \rightarrow \omega_{X / Y}$. By [4], $E$ is semistable on the geometric generic fiber $X_{\bar{\eta}}$ of $f$. By the same idea as in [7], we can apply Bogomolov inequality (Theorem 2.2.1) to $E$. This is however insufficient to get the sharper inequality. Actually we have only Cornalba-Harris-Xiao inequality (cf. Remark 3.10). For the sharper inequality, we need to modify $E$ along singular fibers, namely, we change a compactification of $E_{\bar{\eta}}$ on $X_{\bar{\eta}}$. This modification can be done by a special elementary transformation.

As an application of Theorem 1.1, we can show the following answer for Bogomolov conjecture over function fields (cf. Theorem 5.2). First of all, we fix a notation. Let $\bar{f}: \bar{X} \rightarrow Y$ be the stable model of $f: X \rightarrow Y$. Let $X_{y}$ (resp. $\bar{X}_{y}$ ) be a singular fiber of $f$ (resp. $\bar{f}$ ) over $y$, and $S_{y}$ the set of nodes $P$ of $\bar{X}_{y}$ such that $P$ is not an intersection of two irreducible components of $\bar{X}_{y}$. Let $\pi: Z_{y} \rightarrow \bar{X}_{y}$ be the partial normalization of $\bar{X}_{y}$ at each node in $S_{y}$. We say $X_{y}$ is a chain of stable components if the dual graph of $Z_{y}$ is homeomorphic to the interval $[0,1]$.

Theorem $1.2(\operatorname{char}(k)=0)$. We assume that $f$ is not smooth, every singular fiber of $f$ is a chain of stable components, and one of the following conditions:

(a) the generic fiber of $f$ is hyperelliptic, or

(b) every singular fiber of the stable model $\bar{f}: \bar{X} \rightarrow Y$ has at most one node of type $i>0$. 
Then Bogomolov conjecture holds for the generic fiber of $f$, i.e., we have the following. Let $K$ be the function field of $Y, C$ the generic fiber of $f, \operatorname{Jac}(C)$ the Jacobian of $C$, and let $j: C(\bar{K}) \rightarrow \operatorname{Jac}(C)(\bar{K})$ be a morphism given by $j(P)=\omega_{C}-P$. Then, $j(C(\bar{K}))$ is discrete in terms of the semi-norm arising from the Neron-Tate height paring on $\operatorname{Jac}(C)(\bar{K})$. More precisely, the maximal radius of ball in which we have only finitely many points coming from $C(\bar{K})$ via $j$ is greater than or equal to

$$
\sqrt{\frac{(g-1)^{2}}{g(2 g+1)}\left(\frac{g-1}{3} \delta_{0}+\sum_{i=1}^{\left[\frac{g}{2}\right]} 4 i(g-i) \delta_{i}\right)} .
$$

This is a generalization of the main result of 8 in the case where $\operatorname{char}(k)=0$. For the proof of the above corollary, we will calculate an invariant $e(G, D)$ for a metrized graph $G$ with a polarization $D$. Using this, we have the following inequality, which is rather weaker than the sharper inequality (cf. Corollary 4.6).

Proposition $1.3(\operatorname{char}(k) \geq 0)$. If every singular fiber of $f$ is a chain of stable components, then

$$
\left(\omega_{X / Y} \cdot \omega_{X / Y}\right) \geq \frac{g-1}{3 g} \delta_{0}+\sum_{i=1}^{\left[\frac{g}{2}\right]}\left(\frac{4 i(g-i)}{g}-1\right) \delta_{i} .
$$

Moreover, if the above inequality is strict, then Bogomolov conjecture holds for the generic fiber of $f$.

\section{Preliminaries}

In this section, we will recall base loci of canonical linear systems of reduced curves with only node singularities, and Bogomolov inequality for semistable vector bundles.

2.1. Canonical linear systems of reduced curves with only node singularities. Let $C$ be a reduced projective curve over $k$ with only node singularities. Let $\left\{z_{1}, \ldots, z_{s}\right\}$ be the set of singularities of $C$, and $\pi: \widetilde{C} \rightarrow C$ the normalization of $C$. We set $\pi^{-1}\left(z_{i}\right)=\left\{x_{i}, y_{i}\right\}$ for each $i$. The dualizing sheaf $\omega_{C}$ of $C$ is defined by properties: $(1) \omega_{C} \subset \pi_{*}\left(\Omega_{\widetilde{C}}^{1}\left(\sum_{i} x_{i}+y_{i}\right)\right)$ and (2) for an open set $U$ of $C,\left.t \in \omega_{C}\right|_{U}$ if and only if $\operatorname{Res}_{x_{i}}(t)+\operatorname{Res}_{y_{i}}(t)=0$ for all $i$ with $z_{i} \in U$. Note that $\omega_{C}$ is an invertible sheaf on $C$, i.e., a local section $t \in \pi_{*}\left(\Omega_{\widetilde{C}}^{1}\left(x_{i}+y_{i}\right)\right)_{z_{i}}$ with $\operatorname{Res}_{x_{i}}(t)+\operatorname{Res}_{y_{i}}(t)=0$ and $\operatorname{Res}_{x_{i}}(t) \neq 0$ forms a local frame of $\left(\omega_{C}\right)_{z_{i}}$. The arithmetic genus of $C$, denoted by $p_{a}(C)$, is given by $\operatorname{dim}_{k} H^{0}\left(C, \omega_{C}\right)\left(=\operatorname{dim}_{k} H^{1}\left(C, \mathcal{O}_{C}\right)\right)$.

Lemma 2.1.1 $(\operatorname{char}(k) \geq 0)$. For smooth points $P_{1}, \ldots, P_{n}$ of $C$, let us consider a homomorphism

$$
\phi: H^{0}\left(C, \omega_{C}\left(P_{1}+\cdots+P_{n}\right)\right) \rightarrow k^{n}
$$

defined by $\phi(t)=\left(\operatorname{Res}_{P_{1}}(t), \ldots, \operatorname{Res}_{P_{n}}(t)\right)$. If $C$ is connected, then the kernel of $\phi$ is $H^{0}\left(C, \omega_{C}\right)$ and the image of $\phi$ is the subspace given by $\left\{\left(a_{1}, \ldots, a_{n}\right) \in k^{n} \mid a_{1}+\cdots+a_{n}=0\right\}$. 
Proof. Let $\pi: \widetilde{C} \rightarrow C, x_{i}, y_{i}$ and $z_{i}$ be the same as before. Let $t$ be an element of $H^{0}\left(C, \omega_{C}\left(P_{1}+\cdots+P_{n}\right)\right)$ with $\phi(t)=0$. Then, since $\operatorname{Res}_{P_{j}}(t)=0$ for all $j, t$ has no pole at each $P_{j}$, which implies $t \in H^{0}\left(C, \omega_{C}\right)$. Thus, $\operatorname{Ker}(\phi)=H^{0}\left(C, \omega_{C}\right)$.

Next we would like to see $\sum_{j} \operatorname{Res}_{P_{j}}(t)=0$ for all $t \in H^{0}\left(C, \omega_{C}\left(P_{1}+\cdots+P_{n}\right)\right)$. By the definition of $\omega_{C}, t \in H^{0}\left(\widetilde{C}, \Omega_{\widetilde{C}}^{1}\left(\sum_{i}\left(x_{i}+y_{i}\right)+P_{1}+\cdots+P_{n}\right)\right)$ and $\operatorname{Res}_{x_{i}}(t)+\operatorname{Res}_{y_{i}}(t)=0$ for all $i$. On the other hand, by Residue formula,

$$
\sum_{i=1}^{s}\left(\operatorname{Res}_{x_{i}}(t)+\operatorname{Res}_{y_{i}}(t)\right)+\sum_{j=1}^{n} \operatorname{Res}_{P_{j}}(t)=0 .
$$

Thus, $\sum_{j=1}^{n} \operatorname{Res}_{P_{j}}(t)=0$. Therefore, the image of $\phi$ is contained in $\left\{\left(a_{1}, \ldots, a_{n}\right) \in k^{n} \mid\right.$ $\left.a_{1}+\cdots+a_{n}=0\right\}$.

Since $C$ is connected, by Serre duality,

$$
\operatorname{dim}_{k} H^{1}\left(C, \omega_{C}\left(P_{1}+\cdots+P_{n}\right)\right)=\operatorname{dim}_{k} H^{0}\left(C, \mathcal{O}_{C}\left(-P_{1}-\cdots-P_{n}\right)\right)=0 .
$$

Thus, using Riemann-Roch theorem, we have

$$
\operatorname{dim}_{k} H^{0}\left(C, \omega_{C}\left(P_{1}+\cdots+P_{n}\right)\right)=\operatorname{dim}_{k} H^{0}\left(C, \omega_{C}\right)+n-1 .
$$

Since the kernel of $\phi$ is $H^{0}\left(C, \omega_{C}\right)$, the above formula says us that the dimension of the image of $\phi$ is $n-1$. Therefore, we get the last assertion.

Corollary 2.1.2 $(\operatorname{char}(k) \geq 0)$. Let $C$ be a reduced and irreducible projective curve over $k$ with only node singularities. If $p_{a}(C)>0$, then $\omega_{C}$ is generated by global sections.

Proof. Let $s$ be the number of singularities of $C$ and $g$ the genus of the normalization of $C$. If $s=0$ and $g>0$, or $s=1$ and $g=0$, then our assertion is trivial. We will prove it by induction on $s$. Let $P$ be a node of $C, h: C^{\prime} \rightarrow C$ the partial normalization at $P$, and $h^{-1}(P)=\{Q, R\}$. Then, by hypothesis of induction, $\omega_{C^{\prime}}$ is generated by global sections and $H^{0}\left(C^{\prime} \omega_{C^{\prime}}\right) \subset H^{0}\left(C, \omega_{C}\right)$. Thus, $H^{0}\left(C, \omega_{C}\right) \otimes \mathcal{O}_{C} \rightarrow \omega_{C}$ is surjective outside $P$. On the other hand, by Lemma 2.1.1, there is a section $t$ of $H^{0}\left(C^{\prime}, \omega_{C^{\prime}}(Q+R)\right)$ such that $\operatorname{Res}_{Q}(t)+\operatorname{Res}_{R}(t)=0$ and $\operatorname{Res}_{Q}(t) \neq 0$. By the definition of $\omega_{C}, t \in H^{0}\left(C, \omega_{C}\right)$ and $t$ generates $\left(\omega_{C}\right)_{P}$.

Let $C$ be a connected reduced projective curve over $k$ with only node singularities. Let $P$ be a node of $C$ and $h: C^{\prime} \rightarrow C$ the partial normalization of $C$ at $P$. We say $P$ is a disconnecting node if $C^{\prime}$ is not connected. Note that, if $C$ is semistable curve, then a disconnected node is nothing more than a node of type $i>0$. An irreducible component $D$ of $C$ is said to be of socket type if $D$ is smooth and rational, and all nodes of $C$ on $D$ (i.e. intersections on $D$ with other components) are disconnecting nodes. The base locus of $\left|\omega_{C}\right|$, denoted by $\operatorname{Bs}\left(\omega_{C}\right)$, is defined by the support of $\operatorname{Coker}\left(H^{0}\left(C, \omega_{C}\right) \otimes \mathcal{O}_{C} \rightarrow \omega_{C}\right)$.

Proposition 2.1.3 ( $\operatorname{char}(k) \geq 0)$. Let $C$ be a connected reduced projective curve over $k$ with only node singularities, $D_{1}, \cdots, D_{r}$ irreducible components of socket type, and $D N_{C}$ the set of all disconnecting nodes of $C$. Then we have the following.

(1) $\operatorname{Bs}\left(\omega_{C}\right)=D_{1} \cup \cdots \cup D_{r} \cup D N_{C}$. 
(2) Let $P$ be a disconnecting node of $C, h: C^{\prime} \rightarrow C$ the partial normalization of $C$ at $P$, $C_{1}$ and $C_{2}$ two connected components of $C^{\prime}$. Then,

$$
H^{0}\left(C_{1}, \omega_{C_{1}}\right) \oplus H^{0}\left(C_{2}, \omega_{C_{2}}\right) \stackrel{\sim}{\longrightarrow} H^{0}\left(C, \omega_{C}\right) .
$$

(3) If $P$ is a disconnecting node not lying on irreducible components of socket type, then the image of $H^{0}\left(C, \omega_{C}\right) \otimes \mathcal{O}_{C, P} \rightarrow\left(\omega_{C}\right)_{P}$ coincides with the image of $\left(\Omega_{C}^{1}\right)_{P} \rightarrow\left(\omega_{C}\right)_{P}$.

Proof. In the following, we denote by $N_{C}$ the set of all nodes of $C$. In order to see (1), it is sufficient to show the following facts:

(a) If $P \in N_{C}$ and $P$ is a disconnecting node, then $P \in \operatorname{Bs}\left(\omega_{C}\right)$.

(b) If $P \in N_{C}$ and $P$ is not a disconnecting node, then $P \notin \operatorname{Bs}\left(\omega_{C}\right)$.

(c) If $D$ is an irreducible component of socket type, then $D \subset \operatorname{Bs}\left(\omega_{C}\right)$.

(d) If $D$ is an irreducible component not of socket type, then $H^{0}\left(C, \omega_{C}\right) \otimes \mathcal{O}_{C} \rightarrow \omega_{C}$ is surjective on $D \backslash N_{C}$.

(a) Let $h: C^{\prime} \rightarrow C$ be the partial normalization of $C$ at $P, C_{1}$ and $C_{2}$ two connected components of $C^{\prime}$, and $h^{-1}(P)=\{Q, R\}$ with $Q \in C_{1}$ and $R \in C_{2}$. Let $t$ be any section of $H^{0}\left(C, \omega_{C}\right)$. Then, $\left.t\right|_{C_{1}}$ (resp. $\left.\left.t\right|_{C_{2}}\right)$ is a section of $H^{0}\left(C_{1}, \omega_{C_{1}}(Q)\right)\left(\operatorname{resp} . H^{0}\left(C_{2}, \omega_{C_{2}}(R)\right)\right)$. Then, by Lemma 2.1.1, $\operatorname{Res}_{Q}\left(\left.t\right|_{C_{1}}\right)=\operatorname{Res}_{R}\left(\left.t\right|_{C_{2}}\right)=0$. Thus, $\left.t\right|_{C_{1}} \in H^{0}\left(C_{1}, \omega_{C_{1}}\right)$ and $\left.t\right|_{C_{2}} \in$ $H^{0}\left(C_{2}, \omega_{C_{2}}\right)$. Hence, $t(P)=0$. Therefore, $P \in \operatorname{Bs}\left(\omega_{C}\right)$.

(b) Let $h: C^{\prime} \rightarrow C$ be the partial normalization of $C$ at $P$, and $h^{-1}(P)=\{Q, R\}$. By Lemma 2.1.1, there is a section $t$ of $H^{0}\left(C^{\prime}, \omega_{C^{\prime}}(Q+R)\right)$ such that $\operatorname{Res}_{Q}(t)+\operatorname{Res}_{R}(t)=0$ and $\operatorname{Res}_{Q}(t) \neq 0$. Thus, $t$ is a section of $H^{0}\left(C, \omega_{C}\right)$ and $t$ generates $\left(\omega_{C}\right)_{P}$.

(c) Let $D \cap N_{C}=\left\{Q_{1}, \ldots, Q_{r}\right\}$. Let $t$ be any section of $H^{0}\left(C, \omega_{C}\right)$. Then, $\left.t\right|_{D} \in$ $H^{0}\left(D, \omega_{D}\left(Q_{1}+\cdots+Q_{r}\right)\right)$. Since $Q_{i}$ is a disconnecting node, in the same way as in (a), we can see that $\operatorname{Res}_{Q_{i}}\left(\left.t\right|_{D}\right)=0$ for all $i$. Thus, $\left.t\right|_{D}$ has no pole on $D$. Therefore, $\left.t\right|_{D}=0$ because $D \simeq \mathbb{P}^{1}$. Hence, $D \subset \operatorname{Bs}\left(\omega_{C}\right)$.

(d) If $p_{a}(D)>0$, then our assertion is a consequence of Corollary 2.1.2. Thus, we may assume that $D$ is smooth and rational. Let $E$ be the closure of $C \backslash D$. Since $D$ is not of socket type, there is a connected component $D^{\prime}$ of $E$ with $\#\left(D \cap D^{\prime}\right) \geq 2$. We set $D \cap D^{\prime}=\left\{Q_{1}, \ldots, Q_{r}\right\}(r \geq 2)$ and $D^{\prime \prime}=E \backslash D^{\prime}$. Let $R$ be any point of $D \backslash N_{C}$. We would like to see $R \notin \operatorname{Bs}\left(\omega_{C}\right)$. Since $\omega_{D}\left(Q_{1}+\cdots+Q_{r}\right)$ is generated by global sections, there is a section $t \in H^{0}\left(D, \omega_{D}\left(Q_{1}+\cdots+Q_{r}\right)\right)$ with $t(R) \neq 0$. Since $\operatorname{Res}_{Q_{1}}(t)+\cdots+\operatorname{Res}_{Q_{r}}(t)=0$, by Lemma 2.1.1, there is a section $t^{\prime}$ of $H^{0}\left(D^{\prime}, \omega_{D^{\prime}}\left(Q_{1}+\cdots+Q_{r}\right)\right)$ such that $\operatorname{Res}_{Q_{i}}\left(t^{\prime}\right)+\operatorname{Res}_{Q_{i}}(t)=$ 0 for all $i$. Moreover, let $t^{\prime \prime}$ be the zero form on $D^{\prime \prime}$. Then, $t, t^{\prime}$ and $t^{\prime \prime}$ give a section $s \in H^{0}\left(C, \omega_{C}\right)$ with $\left.s\right|_{D}=t,\left.s\right|_{D^{\prime}}=t^{\prime}$ and $\left.s\right|_{D^{\prime \prime}}=t^{\prime \prime}$. Here $s(R) \neq 0$. Thus, $s$ generates $\left(\omega_{C}\right)_{R}$. Therefore, $R \notin \operatorname{Bs}\left(\omega_{C}\right)$.

(2) is obvious by the proof of (a).

Finally, let us consider (3). Let $h: C^{\prime} \rightarrow C$ be the partial normalization of $C$ at $P$, $C_{1}$ and $C_{2}$ two connected components of $C^{\prime}$, and $h^{-1}(P)=\{Q, R\}$ with $Q \in C_{1}$ and $R \in C_{2}$. Let $x$ (resp. $y$ ) be a local parameter of $C_{1}$ at $Q$ (resp. $C_{2}$ at $R$ ). The image of $H^{0}\left(C, \omega_{C}\right) \otimes \mathcal{O}_{C, P} \rightarrow\left(\omega_{C}\right)_{P}$ is contained in the image of $\left(\Omega_{C}^{1}\right)_{P} \rightarrow\left(\omega_{C}\right)_{P}$ because $H^{0}\left(C, \omega_{C}\right)=$ $H^{0}\left(C_{1}, \omega_{C_{1}}\right) \oplus H^{0}\left(C_{2}, \omega_{C_{2}}\right)$. On the other hand, since $P$ is not lying on irreducible components 
of socket type, there are $t_{1} \in H^{0}\left(C_{1}, \omega_{C_{1}}\right)$ and $t_{2} \in H^{0}\left(C_{2}, \omega_{C_{2}}\right)$ with $t_{1}(Q) \neq 0$ and $t_{2}(R) \neq 0$. Locally, $t_{1}=u_{1} d x$ and $t_{2}=u_{2} d y$, where $u_{1}(Q) \neq 0$ and $u_{2}(R) \neq 0$. Thus, we have (3).

2.2. Bogomolov inequality for fiber spaces. Let $X$ be a smooth projective surface over $k$ and $E$ a torsion free sheaf of rank $r$ on $X$. We set

$$
\delta(E)=2 r c_{2}(E)-(r-1) c_{1}(E)^{2} .
$$

Then we have the following version of Bogomolov inequality for fiber spaces.

Theorem 2.2.1 $(\operatorname{char}(k)=0)$. Let $X$ be a smooth projective surface over $k, Y$ a smooth projective curve over $k$, and $f: X \rightarrow Y$ a surjective morphism with $f_{*}\left(\mathcal{O}_{X}\right)=\mathcal{O}_{Y}$. Let $E$ be a torsion free sheaf on $X$. If $E$ is semistable on the geometric generic fiber $X_{\bar{\eta}}$ of $f$, then $\delta(E) \geq 0$.

Proof. Let $E^{\vee \vee}$ be the double dual of $E$. Then, $E^{\vee \vee}$ is locally free, $c_{1}\left(E^{\vee \vee}\right)=c_{1}(E)$, and $c_{2}\left(E^{\vee \vee}\right)=c_{2}(E)-$ length $\left(E^{\vee \vee} / E\right)$. Thus, we may assume $E$ is locally free.

We assume $\delta(E)<0$. Then, by Bogomolov instability theorem (cf. [1] or [6]), there is a non-zero saturated subsheaf $G$ of $E$ such that, if we set $D=(\operatorname{rk} E) c_{1}(G)-(\operatorname{rk} G) c_{1}(E)$, then $\left(D^{2}\right)>0$ and $(D \cdot H)>0$ for some ample divisor $H$. By Riemann-Roch theorem, we can see that there are a positive number $n$ and an effective divisor $L$ such that $n D$ is linearly equivalent to $L$. Let $F$ be a general fiber of $f$. Then, $(L \cdot F) \geq 0$, which implies that $(D \cdot F) \geq 0$. Here we claim that $(D \cdot F)>0$. To see this, we assume $(D \cdot F)=0$. Then, $(L \cdot F)=0$. Thus, $L$ is a linear combination of irreducible components of fibers. By Zariski lemma, this implies $\left(L^{2}\right) \leq 0$, which contradicts to $\left(D^{2}\right)>0$. Therefore, we get $(D \cdot F)>0$. On the other hand,

$$
(D \cdot F)=(\operatorname{rk} E) \operatorname{deg}\left(\left.G\right|_{F}\right)-(\operatorname{rk} G) \operatorname{deg}\left(\left.E\right|_{F}\right)
$$

Hence, $(D \cdot F)>0$ means that $\left.G\right|_{F}$ is a destabilizing subsheaf of $\left.E\right|_{F}$. This contradicts to the assumption that $E$ is semistable on the geometric generic fiber $X_{\bar{\eta}}$ of $f$.

\section{Proof of Theorem 1.1}

To start the proof of Theorem 1.1, we need a lot of preparations.

Fix an integer $g \geq 2$ and a polynomial $P_{g}(n)=(6 n-1)(g-1)$. Let $H_{g} \subset \operatorname{Hilb}_{\mathbb{P} 5 g-6}^{P_{g}}$ be a subscheme of all tri-canonically embededded stable curves over $k, Z_{g} \subset H_{g} \times \mathbb{P}^{5 g-6}$ the universal tri-canonically embededded stable curves over $k$, and $\pi: Z_{g} \rightarrow H_{g}$ the natural projection. Let $S$ be the set of all points $x \in Z_{g}$ such that $\pi$ is not smooth at $x$, and $\Delta=\pi(S)$. Then, by [3, Theorem (1.6) and Corollary (1.9)], $Z_{g}, H_{g}$, and $S$ are smooth over $k, \Delta$ and $\pi^{*}(\Delta)$ are divisors with only normal crossings, and $\left.\pi\right|_{S}: S \rightarrow \Delta$ is the normalization of $\Delta$. Let $\Delta=\Delta_{0} \cup \cdots \cup \Delta_{[g / 2]}$ be the irreducible decomposition of $\Delta$ such that, if $x \in \Delta_{i} \backslash \operatorname{Sing}(\Delta)$, then $\pi^{-1}(x)$ is a stable curve with one node of type $i$. Let $\delta_{i}(x)$ be the number of nodes of type $i$ on a fiber $\pi^{-1}(x)$ over $x \in Z_{g}$. Since $\delta_{i}(x)=\operatorname{mult}_{x}\left(\Delta_{i}\right)$, $\delta_{i}: H_{g} \rightarrow \mathbb{Z}$ is upper-semicontinuous. Therefore, if we set

$$
H_{g}^{0}=\left\{x \in H_{g} \mid \pi^{-1}(x) \text { has at most one node of type } i>0\right\},
$$

then $H_{g}^{0}$ is an open set. In other words, $H_{g}^{0}=H_{g} \backslash \operatorname{Sing}\left(\Delta_{1}+\cdots+\Delta_{[g / 2]}\right)$. We set $Z_{g}^{0}=\pi^{-1}\left(H_{g}^{0}\right), \Delta^{0}=\Delta \cap H_{g}^{0}, \Delta_{i}^{0}=\Delta_{i} \cap H_{g}^{0}, S^{0}=\left(\left.\pi\right|_{S}\right)^{-1}\left(\Delta^{0}\right)$, and $S_{i}^{0}=\left(\left.\pi\right|_{S}\right)^{-1}\left(\Delta_{i}^{0}\right)$. 
Then, for all $1 \leq i<j \leq[g / 2], \Delta_{i}^{0} \cap \Delta_{j}^{0}=\emptyset$. For $1 \leq i \leq[g / 2]$, let $\pi^{-1}\left(\Delta_{i}^{0}\right)=C_{i}^{1} \cup C_{i}^{2}$ be the irreducible decomposition such that the generic fiber of $\left.\pi\right|_{C_{i}^{1}}: C_{i}^{1} \rightarrow \Delta_{i}^{0}$ (resp. $\left.\pi\right|_{C_{i}^{2}}: C_{i}^{2} \rightarrow \Delta_{i}^{0}$ ) is of genus $i$ (resp. $g-i$ ). Then, $C_{i}^{1} \cap C_{i}^{2}=S_{i}^{0}$. Moreover, we denote $S_{1}^{0} \cup \cdots \cup S_{[g / 2]}^{0}$ by $S_{+}^{0}$.

Claim 3.1. $\operatorname{Supp}\left(\operatorname{Coker}\left(\pi^{*} \pi_{*}\left(\omega_{Z_{g}^{0} / H_{g}^{0}}\right) \rightarrow \omega_{Z_{g}^{0} / H_{g}^{0}}\right)\right)=S_{+}^{0}$ and, for $z \in S_{+}^{0}$, the image of $\left(\pi^{*} \pi_{*}\left(\omega_{Z_{g}^{0} / H_{g}^{0}}\right)\right)_{z} \rightarrow\left(\omega_{Z_{g}^{0} / H_{g}^{0}}\right)_{z}$ is $\left(\Omega_{Z_{g}^{0} / H_{g}^{0}}^{1}\right)_{z}$.

Proof. The first assertion is a consequence of (1) of Proposition 2.1.3. Let $F$ be the image of $\pi^{*} \pi_{*}\left(\omega_{Z_{g}^{0} / H_{g}^{0}}\right) \rightarrow \omega_{Z_{g}^{0} / H_{g}^{0}}$ and $x=\pi(z)$. As we see in (2) of Proposition 2.1.3, any section $s$ of $\left(\pi_{*}\left(\omega_{Z_{g}^{0} / H_{g}^{0}}\right)\right)_{x}$ has no pole on irreducible components of fibers around $z$, which means $F_{z} \subseteq\left(\Omega_{Z_{g}^{0} / H_{g}^{0}}^{1}\right)_{z}$. Therefore, by (3) of Proposition 2.1.3, we can conclude $F_{z}=\left(\Omega_{Z_{g}^{0} / H_{g}^{0}}^{1}\right)_{z_{\square}}$.

We set

$$
E=\operatorname{Ker}\left(\pi^{*} \pi_{*}\left(\omega_{Z_{g}^{0} / H_{g}^{0}}\right) \longrightarrow \omega_{Z_{g}^{0} / H_{g}^{0}}\right) .
$$

Claim 3.2. E is locally free.

Proof. Let $I_{S^{0}}$ (resp. $I_{S_{+}^{0}}$ ) be the defining ideal of $S^{0}$ (resp. $S_{+}^{0}$ ). It is well known that $\Omega_{Z_{g}^{0} / H_{g}^{0}}^{1}=I_{S^{0}} \cdot \omega_{Z_{g}^{0} / H_{g}^{0}}$ (cf. [10, Proof of Theorem 5.10]). Thus, by Claim 3.1, the image of $\pi^{*} \pi_{*}\left(\omega_{Z_{g}^{0} / H_{g}^{0}}\right) \rightarrow \omega_{Z_{g}^{0} / H_{g}^{0}}$ is $I_{S_{+}^{0}} \cdot \omega_{Z_{g}^{0} / H_{g}^{0}}$. Since $S_{+}^{0}$ is smooth and of codimension 2, $\mathcal{E}_{X} t_{\mathcal{O}_{Z_{g}^{0}}}^{i}\left(I_{S_{+}^{0}}, \mathcal{O}_{Z_{g}^{0}}\right)=0$ for $i \geq 2$. Therefore, we can see that $\mathcal{E}_{X} t_{\mathcal{O}_{Z_{g}^{0}}}^{i}\left(E, \mathcal{O}_{Z_{g}^{0}}\right)=0$ for $i \geq 1$. Hence, we have our claim.

Claim 3.3. For $1 \leq i \leq[g / 2], \pi_{*}\left(\omega_{C_{i}^{1} / \Delta_{i}^{0}}\right) \oplus \pi_{*}\left(\omega_{C_{i}^{2} / \Delta_{i}^{0}}\right) \stackrel{\sim}{\longrightarrow} \pi_{*}\left(\omega_{C_{i}^{1} \cup C_{i}^{2} / \Delta_{i}^{0}}\right)$.

Proof. Clearly we have the natural injection

$$
\pi_{*}\left(\omega_{C_{i}^{1} / \Delta_{i}^{0}}\right) \oplus \pi_{*}\left(\omega_{C_{i}^{2} / \Delta_{i}^{0}}\right) \longrightarrow \pi_{*}\left(\omega_{C_{i}^{1} \cup C_{i}^{2} / \Delta_{i}^{0}}\right) .
$$

For $x \in \Delta_{i}^{0}$, if we set $C_{1}=\pi^{-1}(x) \cap C_{i}^{1}$ and $C_{2}=\pi^{-1}(x) \cap C_{i}^{2}$, then by (2) of Proposition 2.1.3

$$
H^{0}\left(C_{1}, \omega_{C_{1}}\right) \oplus H^{0}\left(C_{2}, \omega_{C_{2}}\right)=H^{0}\left(\pi^{-1}(x), \omega_{\pi^{-1}(x)}\right) .
$$

Thus, the above injection is bijective.

For $1 \leq i \leq[g / 2]$ and $j=1,2$, let

$$
Q_{i}^{j}=\operatorname{Ker}\left(\left(\left.\pi\right|_{C_{i}^{j}}\right)^{*}\left(\left.\pi\right|_{C_{i}^{j}}\right)_{*}\left(\omega_{C_{i}^{j} / \Delta_{i}^{0}}\right) \longrightarrow \omega_{C_{i}^{j} / \Delta_{i}^{0}}\right) \text {. }
$$

Claim 3.4. $Q_{i}^{j}$ is a locally free sheaf on $C_{i}^{j}$.

Proof. The homomorphism $\left(\left.\pi\right|_{C_{i}^{j}}\right)^{*}\left(\left.\pi\right|_{C_{i}^{j}}\right)_{*}\left(\omega_{C_{i}^{j} / \Delta_{i}^{0}}\right) \rightarrow \omega_{C_{i}^{j} / \Delta_{i}^{0}}$ is surjective by (1) of Proposition 2.1.3. Therefore, we can see our claim. 
Using projection $\pi_{*}\left(\omega_{C_{i}^{1} \cup C_{i}^{2} / \Delta_{i}^{0}}\right) \rightarrow \pi_{*}\left(\omega_{C_{i}^{j} / \Delta_{i}^{0}}\right)$, we have the following commutative diagram:

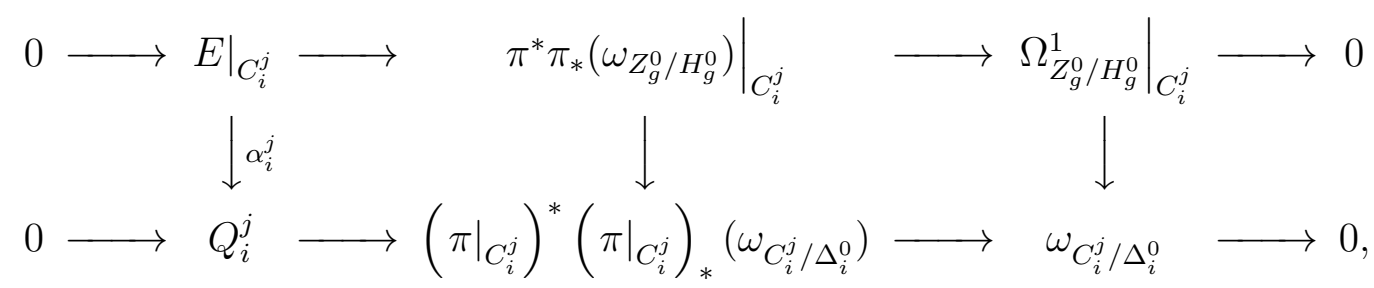

where $\alpha_{i}^{j}:\left.E\right|_{C_{i}^{j}} \rightarrow Q_{i}^{j}$ is the induced homomorphism. Thus, we can give a homomorphism

$$
\phi_{i}:\left.\left.E \rightarrow E\right|_{C_{i}^{1}} \oplus E\right|_{C_{i}^{2}} \stackrel{\alpha_{i}^{1} \oplus \alpha_{i}^{2}}{\longrightarrow} Q_{i}^{1} \oplus Q_{i}^{2}
$$

Claim 3.5. $\phi_{i}$ is surjective.

Proof. To show this claim, it is sufficient to see it on each fiber. Let $C$ be a fiber of $\pi$ over $x \in \Delta_{i}^{0}$, and $C=C^{1} \cup C^{2}$ the irreducible decomposition with $C^{1}=C \cap C_{i}^{1}$ and $C^{2}=C \cap C_{i}^{2}$. For simplicity, we set $E_{C}=\left.E\right|_{C}, Q^{1}=\left.Q_{i}^{1}\right|_{C^{1}}, Q^{2}=\left.Q_{i}^{2}\right|_{C^{2}}$, i.e., $E_{C}$ is the kernel of $H^{0}\left(C, \omega_{C}\right) \otimes \mathcal{O}_{C} \rightarrow \omega_{C}$ and $Q^{j}$ is the kernel of $H^{0}\left(C^{j}, \omega_{C^{j}}\right) \otimes \mathcal{O}_{C^{j}} \rightarrow \omega_{C^{j}}$. Since $H^{0}\left(C, \omega_{C}\right)=H^{0}\left(C^{1}, \omega_{C^{1}}\right) \oplus H^{0}\left(C^{2}, \omega_{C^{2}}\right), Q^{1} \oplus Q^{2} \subset H^{0}\left(C, \omega_{C}\right) \otimes \mathcal{O}_{C}$. On the other hand, $Q^{1}, Q^{2} \subset E_{C}$. Thus, we get $Q^{1} \oplus Q^{2} \subset E_{C}$, which shows us that $E_{C} \rightarrow Q^{1} \oplus Q^{2}$ is surjective.

Let

$$
F=\operatorname{Ker}\left(\bigoplus_{i=1}^{\left[\frac{g}{2}\right]} \phi_{i}: E \longrightarrow \bigoplus_{i=1}^{\left[\frac{g}{2}\right]} Q_{i}^{1} \oplus Q_{i}^{2}\right)
$$

Claim 3.6. $F$ is locally free.

Proof. We set $F_{1}=\operatorname{Ker}\left(E \rightarrow \bigoplus_{i=1}^{[g / 2]} Q_{i}^{1}\right)$. Then, $F=\operatorname{Ker}\left(F_{1} \rightarrow \bigoplus_{i=1}^{[g / 2]} Q_{i}^{2}\right) . F_{1}$ is an elementary transformation of $E$. Thus, by [5], $F_{1}$ is locally free. Since $\phi_{i}$ is surjective, so is $\left.F_{1}\right|_{C_{i}^{2}} \rightarrow Q_{i}^{2}$. Therefore, $F$ is locally free because $F$ is an elementary transformation of $F_{1}$.

For a vector bundle $G$ on $Z_{g}^{0}$, we define $\delta(G) \in A_{\operatorname{dim} H_{g}^{0}-1}\left(H_{g}^{0}\right)$ by

$$
\delta(G)=\pi_{*}\left(2 \operatorname{rk}(G) c_{2}(G)-(\operatorname{rk}(G)-1) c_{1}(G)^{2}\right) .
$$

Claim 3.7. $\delta(E)=(8 g+4) c_{1}\left(\pi_{*}\left(\omega_{Z_{g}^{0} / H_{g}^{0}}\right)\right)-g \Delta_{0}^{0}-(3 g-2) \sum_{i=1}^{\left[\frac{g}{2}\right]} \Delta_{i}^{0}$.

Proof. First of all, let us recall the Grothendieck-Riemann-Roch theorem. Let $f: X \rightarrow Y$ be a proper morphism of smooth varieties over $k$. The Grothendieck-Riemann-Roch theorem says that, for any coherent sheaf $\mathcal{F}$ on $X$,

$$
\operatorname{ch}\left(\sum_{i}(-1)^{i} R^{i} f_{*}(\mathcal{F})\right)=f_{*}\left(\operatorname{ch}(\mathcal{F}) \operatorname{td}\left(T_{X / Y}\right)\right) .
$$


We denote $\operatorname{ch}\left(\sum_{i} R^{i} f_{*}(\mathcal{F})\right)$ by $\chi(X / Y, \mathcal{F})$, and $A_{i}(X)$-component of $f_{*}\left(\operatorname{ch}(\mathcal{F}) \operatorname{td}\left(T_{X / Y}\right)\right)$ by $f_{*}\left(\operatorname{ch}(\mathcal{F}) \operatorname{td}\left(T_{X / Y}\right)\right)_{(i)}$. Note that if $\operatorname{dim}(X / Y)=1$, then

$$
f_{*}\left(\operatorname{ch}(\mathcal{F}) \operatorname{td}\left(T_{X / Y}\right)\right)_{(\operatorname{dim} Y)}=f_{*}\left(c_{1}(\mathcal{F})-\operatorname{rk}(\mathcal{F}) \frac{\omega_{X / Y}}{2}\right)
$$

and

$$
\begin{aligned}
& f_{*}\left(\operatorname{ch}(\mathcal{F}) \operatorname{td}\left(T_{X / Y}\right)\right)_{(\operatorname{dim} Y-1)}= \\
& f_{*}\left(\frac{c_{1}(\mathcal{F}) \cdot\left(c_{1}(\mathcal{F})-\omega_{X / Y}\right)}{2}-c_{2}(\mathcal{F})+\operatorname{rk}(\mathcal{F}) \frac{\omega_{X / Y}^{2}+c_{2}\left(\Omega_{X / Y}^{1}\right)}{12}\right) .
\end{aligned}
$$

Let us go back to the proof of our claim. For simplicity, we denote $c_{1}\left(\pi_{*}\left(\omega_{Z_{g}^{0} / H_{g}^{0}}\right)\right)$ and $\omega_{Z_{g}^{0} / H_{g}^{0}}$ by $\lambda$ and $\omega$ respectively. Consider an exact sequence

$$
0 \rightarrow E \rightarrow \pi^{*}\left(\pi_{*}(\omega)\right) \rightarrow I_{S_{+}^{0}} \cdot \omega \rightarrow 0
$$

First of all, we have $c_{1}(E)=\pi^{*}(\lambda)-\omega$. Moreover,

$$
\chi\left(Z_{g}^{0} / H_{g}^{0}, \pi^{*}\left(\pi_{*}(\omega)\right)\right)=\chi\left(Z_{g}^{0} / H_{g}^{0}, E\right)+\chi\left(Z_{g}^{0} / H_{g}^{0}, I_{S_{+}^{0}} \cdot \omega\right) .
$$

Thus, using the Grothendieck-Riemann-Roch theorem, we can see

$$
\pi_{*}\left(c_{2}(E)\right)=\pi_{*}\left(\omega \cdot \omega-\pi^{*}(\lambda) \cdot \omega-S_{+}^{0}+c_{2}\left(\pi^{*}\left(\pi_{*}(\omega)\right)\right)\right) .
$$

Noting that $\pi_{*}\left(\pi^{*}(\lambda) \cdot \omega\right)=(2 g-2) \lambda, \pi_{*}\left(c_{2}\left(\pi^{*}\left(\pi_{*}(\omega)\right)\right)\right)=0$ and $\pi_{*}\left(S_{i}^{0}\right)=\Delta_{i}^{0}$, the above implies

$$
\pi_{*}\left(c_{2}(E)\right)=\pi_{*}(\omega \cdot \omega)-(2 g-2) \lambda-\sum_{i=1}^{\left[\frac{g}{2}\right]} \Delta_{i}^{0} .
$$

Therefore, by virtue of Noether formula: $\pi_{*}(\omega \cdot \omega)=12 \lambda-\Delta^{0}$, we can conclude our claim.

Claim 3.8. $\delta(F)=(8 g+4) c_{1}\left(\pi_{*}\left(\omega_{Z_{g}^{0} / H_{g}^{0}}\right)\right)-g \Delta_{0}^{0}-\sum_{i=1}^{\left[\frac{g}{2}\right]} 4 i(g-i) \Delta_{i}^{0}$.

Proof. Consider an exact sequence:

$$
0 \rightarrow F \rightarrow E \rightarrow \bigoplus_{i=1}^{\left[\frac{g}{2}\right]}\left(Q_{i}^{1} \oplus Q_{i}^{2}\right) \rightarrow 0
$$

First of all, we have

$$
\begin{aligned}
c_{1}(F) & =c_{1}(E)-\sum_{i=1}^{\left[\frac{g}{2}\right]}\left(\operatorname{rk}\left(Q_{i}^{1}\right) C_{i}^{1}+\operatorname{rk}\left(Q_{i}^{2}\right) C_{i}^{2}\right) \\
& =c_{1}(E)-\sum_{i=1}^{\left[\frac{g}{2}\right]}\left((i-1) C_{i}^{1}+\left(i^{\prime}-1\right) C_{i}^{2}\right),
\end{aligned}
$$


where $i^{\prime}=g-i$. Moreover, the above exact sequence gives rise to

$$
\chi\left(Z_{g}^{0} / H_{g}^{0}, E\right)=\chi\left(Z_{g}^{0} / H_{g}^{0}, F\right)+\sum_{i=1}^{\left[\frac{g}{2}\right]}\left(\chi\left(C_{i}^{1} / \Delta_{i}^{0}, Q_{i}^{1}\right)+\chi\left(C_{i}^{2} / \Delta_{i}^{0}, Q_{i}^{2}\right)\right),
$$

which, by Grothendieck-Riemann-Roch theorem, implies

$$
\begin{aligned}
& \pi_{*}\left(c_{2}(F)\right)=\pi_{*}\left(c_{2}(E)+\frac{c_{1}(F) \cdot\left(c_{1}(F)-\omega\right)}{2}-\frac{c_{1}(E) \cdot\left(c_{1}(E)-\omega\right)}{2}\right)+ \\
& \sum_{i=1}^{\left[\frac{g}{2}\right]}\left\{\left(\left.\pi\right|_{C_{i}^{1}}\right)_{*}\left(c_{1}\left(Q_{i}^{1}\right)-\frac{(i-1) \omega_{C_{i}^{1} / \Delta_{i}^{0}}}{2}\right)+\left(\left.\pi\right|_{C_{i}^{2}}\right)_{*}\left(c_{1}\left(Q_{i}^{2}\right)-\frac{\left.\left.\left(i^{\prime}-1\right) \omega_{C_{i}^{2} / \Delta_{i}^{0}}\right)\right\} .}{2}\right.\right.
\end{aligned}
$$

Thus, we have

$$
\begin{aligned}
\delta(F)=\delta(E)+ & \sum_{i=1}^{\left[\frac{g}{2}\right]} \pi_{*}\left(\left((i-1) C_{i}^{1}+\left(i^{\prime}-1\right) C_{i}^{2}\right)^{2}\right) \\
& +\sum_{i=1}^{\left[\frac{g}{2}\right]} \pi_{*}\left(\left((g-1) \omega-2 c_{1}(E)\right) \cdot\left((i-1) C_{i}^{1}+\left(i^{\prime}-1\right) C_{i}^{2}\right)\right) \\
& +2(g-1) \sum_{i=1}^{\left[\frac{g}{2}\right]}\left(\left.\pi\right|_{C_{i}^{1}}\right)_{*}\left(c_{1}\left(Q_{i}^{1}\right)-\frac{(i-1) \omega_{C_{i}^{1} / \Delta_{i}^{0}}}{2}\right) \\
& \quad+2(g-1) \sum_{i=1}^{\left[\frac{g}{2}\right]}\left(\left.\pi\right|_{C_{i}^{2}}\right)_{*}\left(c_{1}\left(Q_{i}^{2}\right)-\frac{\left(i^{\prime}-1\right) \omega_{C_{i}^{2} / \Delta_{i}^{0}}}{2}\right) .
\end{aligned}
$$

Therefore, using formulae:

$$
\begin{cases}\left(\left.\pi\right|_{C_{i}^{1}}\right)_{*}\left(c_{1}\left(Q_{i}^{1}\right)\right)=-2(i-1) \Delta_{i}^{0}, & \left(\left.\pi\right|_{C_{i}^{2}}\right)_{*}\left(c_{1}\left(Q_{i}^{2}\right)\right)=-2\left(i^{\prime}-1\right) \Delta_{i}^{0}, \\ \left(\left.\pi\right|_{C_{i}^{1}}\right)_{*}\left(\omega_{C_{i}^{1} / \Delta_{i}^{0}}\right)=2(i-1) \Delta_{i}^{0}, & \left(\left.\pi\right|_{C_{i}^{2}}\right)_{*}\left(\omega_{C_{i}^{2} / \Delta_{i}^{0}}\right)=2\left(i^{\prime}-1\right) \Delta_{i}^{0}, \\ \pi_{*}\left(c_{1}(E) \cdot C_{i}^{1}\right)=-(2(i-1)+1) \Delta_{i}^{0}, & \pi_{*}\left(c_{1}(E) \cdot C_{i}^{2}\right)=-\left(2\left(i^{\prime}-1\right)+1\right) \Delta_{i}^{0}, \\ \pi_{*}\left(\omega \cdot C_{i}^{1}\right)=(2(i-1)+1) \Delta_{i}^{0}, & \pi_{*}\left(\omega \cdot C_{i}^{2}\right)=\left(2\left(i^{\prime}-1\right)+1\right) \Delta_{i}^{0}, \\ \pi_{*}\left(C_{i}^{1} \cdot C_{i}^{2}\right)=\Delta_{i}^{0}, & \pi_{*}\left(C_{i}^{j} \cdot C_{i}^{j}\right)=-\Delta_{i}^{0} \quad \text { for } j=1,2,\end{cases}
$$

we can see that

$$
\delta(F)=\delta(E)+\sum_{i=1}^{\left[\frac{g}{2}\right]}\left(3 g-2-4 i i^{\prime}\right) \Delta_{i}^{0}
$$


Hence, by Claim 3.7, we get

$$
\delta(F)=(8 g+4) c_{1}\left(\pi_{*}\left(\omega_{Z_{g}^{0} / H_{g}^{0}}\right)\right)-g \Delta_{0}^{0}-\sum_{i=1}^{\left[\frac{g}{2}\right]} 4 i(g-i) \Delta_{i}^{0} .
$$

Proof of Theorem 1.1: $\quad$ Now we obtain everything to prove Theorem 1.1. Let $f: X \rightarrow$ $Y$ be a semistable curve as in Theorem 1.1. Then, there is a morphism $h: Y \rightarrow H_{g}^{0}$ with $Z_{g}^{0} \times_{H_{g}^{0}} Y \simeq \bar{X}$. Let $h^{\prime}: X \rightarrow Z_{g}^{0}$ be the induced morphism. Let us consider a vector bundle $h^{\prime *}(F)$ on $Y$. By [4], $h^{\prime *}(F)$ is semistable on the generic fiber of $f$. Thus, $\delta\left(h^{\prime *}(F)\right) \geq 0$ by Theorem 2.2.1. On the other hand, by Claim 3.8,

$$
\begin{aligned}
\delta\left(h^{\prime *}(F)\right) & =\operatorname{deg}\left(h^{*}\left((8 g+4) c_{1}\left(\pi_{*}\left(\omega_{Z_{g}^{0} / H_{g}^{0}}\right)\right)-g \Delta_{0}^{0}-\sum_{i=1}^{\left[\frac{g}{2}\right]} 4 i(g-i) \Delta_{i}^{0}\right)\right) \\
& =(8 g+4) \operatorname{deg}\left(f_{*}\left(\omega_{X / Y}\right)\right)-g \delta_{0}-\sum_{i=1}^{\left[\frac{g}{2}\right]} 4 i(g-i) \delta_{i} .
\end{aligned}
$$

Thus, we have

$$
(8 g+4) \operatorname{deg}\left(f_{*}\left(\omega_{X / Y}\right)\right) \geq g \delta_{0}+\sum_{i=1}^{\left[\frac{g}{2}\right]} 4 i(g-i) \delta_{i} .
$$

Let $\iota: Z_{g}^{0} \rightarrow Z_{g}$ be the inclusion map. If we set $\bar{F}=\iota_{*}(F)$, then $\bar{F}$ is a reflexive coherent sheaf on $Z_{g}$ because $\operatorname{codim}\left(Z_{g} \backslash Z_{g}^{0}\right)=2$. Using $\bar{F}$, we can slightly generalize Theorem 1.1 .

Theorem $3.9(\operatorname{char}(k)=0)$. Let $X$ be a smooth projective surface over $k, Y$ a smooth projective curve over $k$, and $f: X \rightarrow Y$ a semistable curve of genus $g \geq 2$ over $Y$. Let $h: Y \rightarrow H_{g}$ and $h^{\prime}: X \rightarrow Z_{g}$ be the induced morphisms such that the following diagram is commutative:

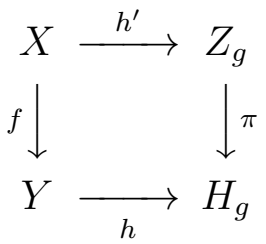

If $\bar{F}$ is locally free along $h^{\prime}(X)$, then

$$
(8 g+4) \operatorname{deg}\left(f_{*}\left(\omega_{X / Y}\right)\right) \geq g \delta_{0}+\sum_{i=1}^{\left[\frac{g}{2}\right]} 4 i(g-i) \delta_{i} .
$$

Proof. Since $\operatorname{codim}\left(H_{g} \backslash H_{g}^{0}\right)=2$, we have

$$
\delta(\bar{F})=(8 g+4) c_{1}\left(\pi_{*}\left(\omega_{Z_{g} / H_{g}}\right)\right)-g \Delta_{0}-\sum_{i=1}^{\left[\frac{g}{2}\right]} 4 i(g-i) \Delta_{i} .
$$


Thus, we can conclude our theorem in the same way as before.

Remark 3.10. Let $X$ be a smooth projective surface over $k, Y$ a smooth projective curve over $k$, and $f: X \rightarrow Y$ a surjective morphism with $f_{*}\left(\mathcal{O}_{X}\right)=\mathcal{O}_{Y}$. Then, in the same idea of Claim 3.7 or [7], we can see that, if $E$ is the kernel of $f^{*} f_{*}\left(\omega_{X / Y}\right) \rightarrow \omega_{X / Y}$ and $\omega_{X / Y}$ is $f$-nef,

$$
\delta(E) \leq g\left(\omega_{X / Y} \cdot \omega_{X / Y}\right)-4(g-1) \operatorname{deg}\left(f_{*}\left(\omega_{X / Y}\right)\right),
$$

where $g \geq 2$ is the genus of the generic fiber. Thus, by [4] and Theorem 2.2.1, we can recover Cornalba-Harris-Xiao inequality:

$$
g\left(\omega_{X / Y} \cdot \omega_{X / Y}\right) \geq 4(g-1) \operatorname{deg}\left(f_{*}\left(\omega_{X / Y}\right)\right) .
$$

\section{Calculation of invariants arising from Green functions}

In this section, we would like to calculate an invariant $e(G, D)$ for a metrized graph $G$ with a polarization $D$. For details of metrized graphs, see 12.

Let $G$ be a connected metrized graph and $D$ an $\mathbb{R}$-divisor on $G$. If $\operatorname{deg}(D) \neq-2$, then there are a unique measure $\mu_{(G, D)}$ on $G$ and a unique function $g_{(G, D)}$ on $G \times G$ with the following properties.

(a) $\int_{G} \mu_{(G, D)}=1$.

(b) $g_{(G, D)}(x, y)$ is symmetric and continuous on $G \times G$.

(c) For a fixed $x \in G, \Delta_{y}\left(g_{(G, D)}(x, y)\right)=\delta_{x}-\mu_{(G, D)}$.

(d) For a fixed $x \in G, \int_{G} g_{(G, D)}(x, y) \mu_{(G, D)}(y)=0$.

(e) $g_{(G, D)}(D, y)+g_{(G, D)}(y, y)$ is a constant for all $y \in G$.

The constant $g_{(G, D)}(D, y)+g_{(G, D)}(y, y)$ is denoted by $c(G, D)$. Further we set

$$
e(G, D)=2 \operatorname{deg}(D) c(G, D)-g_{(G, D)}(D, D) .
$$

First of all, let's consider another expression of $e(G, D)$.

Lemma 4.1. Let $G$ be a connected metrized graph and $D$ an $\mathbb{R}$-divisor on $G$ with $\operatorname{deg}(D) \neq$ -2 . Then, for any point $O$ of $G$,

$$
e(G, D)=(\operatorname{deg}(D)+2) g_{(G, D)}(O, D)+r_{G}(O, D),
$$

where $r_{G}(P, Q)$ is the resistance between $P$ and $Q$ on $G$.

Proof. We set $D=\sum_{i} a_{i} P_{i}$. Then,

$$
\begin{aligned}
e(G, D) & =2 \operatorname{deg}(D) c(G, D)-\sum_{i} a_{i} g_{(G, D)}\left(D, P_{i}\right) \\
& =2 \operatorname{deg}(D) c(G, D)-\sum_{i} a_{i}\left(c(G, D)-g_{(G, D)}\left(P_{i}, P_{i}\right)\right) \\
& =\operatorname{deg}(D) c(G, D)+\sum_{i} a_{i} g_{(G, D)}\left(P_{i}, P_{i}\right)
\end{aligned}
$$


Since we know

$$
r_{G}(P, Q)=g_{(G, D)}(P, P)-2 g_{(G, D)}(P, Q)+g_{(G, D)}(Q, Q)
$$

for all points $P, Q \in G$, the above implies

$$
\begin{aligned}
e(G, D)= & \operatorname{deg}(D)\left(g_{(G, D)}(O, O)+g_{(G, D)}(O, D)\right) \\
& +\sum_{i} a_{i}\left(r_{G}\left(O, P_{i}\right)+2 g_{(G, D)}\left(O, P_{i}\right)-g_{(G, D)}(O, O)\right) \\
= & (\operatorname{deg}(D)+2) g_{(G, D)}(O, D)+r_{G}(O, D) .
\end{aligned}
$$

Let $G_{1}$ and $G_{2}$ be metrized graphs. Fix points $x_{1} \in G_{1}$ and $x_{2} \in G_{2}$. The one point sum $G_{1} \vee G_{2}$ with respect to $x_{1}$ and $x_{2}$, defined by $G_{1} \times\left\{x_{2}\right\} \cup\left\{x_{1}\right\} \times G_{2}$ in $G_{1} \times G_{2}$, is a metrized graph obtained by joining $x_{1} \in G_{1}$ and $x_{2} \in G_{2}$. The joining point, which is $\left\{x_{1}\right\} \times\left\{x_{2}\right\}$ in $G_{1} \times G_{2}$, is denoted by $j\left(G_{1} \vee G_{2}\right)$. Any $\mathbb{R}$-divisor on $G_{i}(i=1,2)$ can be viewed as an $\mathbb{R}$-divisor on $G_{1} \vee G_{2}$.

Proposition 4.2. Let $G_{1}$ and $G_{2}$ be connected metrized graphs, and $D_{1}$ and $D_{2} \mathbb{R}$-divisors on $G_{1}$ and $G_{2}$ respectively with $\operatorname{deg}\left(D_{i}\right) \neq-2(i=1,2)$. Let $G=G_{1} \vee G_{2}, O=j\left(G_{1} \vee G_{2}\right)$, and $D=D_{1}+D_{2}$ on $G_{1} \vee G_{2}$. If $\operatorname{deg}\left(D_{1}+D_{2}\right) \neq-2$, then

$$
\begin{aligned}
e(G, D) & =e\left(G_{1}, D_{1}\right)+e\left(G_{2}, D_{2}\right) \\
& +\frac{2 \operatorname{deg}\left(D_{2}\right)\left(\operatorname{deg}\left(D_{1}\right)+2\right) g_{\left(G_{1}, D_{1}\right)}(O, O)+2 \operatorname{deg}\left(D_{1}\right)\left(\operatorname{deg}\left(D_{2}\right)+2\right) g_{\left(G_{2}, D_{2}\right)}(O, O)}{\operatorname{deg}\left(D_{1}\right)+\operatorname{deg}\left(D_{2}\right)+2} .
\end{aligned}
$$

Moreover, if $P \in G_{2}$, then

$$
\begin{aligned}
g_{(G, D)}(P, P)= & \frac{\operatorname{deg}\left(D_{1}\right)}{\operatorname{deg}\left(D_{1}\right)+\operatorname{deg}\left(D_{2}\right)+2} r_{G_{2}}(O, P) \\
& +\frac{\operatorname{deg}\left(D_{2}\right)+2}{\operatorname{deg}\left(D_{1}\right)+\operatorname{deg}\left(D_{2}\right)+2} g_{\left(G_{2}, D_{2}\right)}(P, P) \\
& -\frac{\operatorname{deg}\left(D_{1}\right)\left(\operatorname{deg}\left(D_{2}\right)+2\right)}{\left(\operatorname{deg}\left(D_{1}\right)+\operatorname{deg}\left(D_{2}\right)+2\right)^{2}} g_{\left(G_{2}, D_{2}\right)}(O, O) \\
& +\frac{\left(\operatorname{deg}\left(D_{1}\right)+2\right)^{2}}{\left(\operatorname{deg}\left(D_{1}\right)+\operatorname{deg}\left(D_{2}\right)+2\right)^{2}} g_{\left(G_{1}, D_{1}\right)}(O, O) .
\end{aligned}
$$

Proof. For simplicity, we set $d_{i}=\operatorname{deg}\left(D_{i}\right)$ and $g_{i}=g_{\left(G_{i}, D_{i}\right)}(O, O)$ for $i=1,2$. By $[12$, Lemma 3.7], we have

$$
\mu_{(G, D)}=\frac{d_{1}+2}{d_{1}+d_{2}+2} \mu_{\left(G_{1}, D_{1}\right)}+\frac{d_{2}+2}{d_{1}+d_{2}+2} \mu_{\left(G_{2}, D_{2}\right)}-\frac{2}{d_{1}+d_{2}+2} \delta_{O} .
$$


Consider the following function on $G$ :

$$
g(x)= \begin{cases}\frac{d_{1}+2}{d_{1}+d_{2}+2} g_{\left(G_{1}, D_{1}\right)}(O, x)+\frac{\left(d_{2}+2\right)^{2} g_{2}-d_{2}\left(d_{1}+2\right) g_{1}}{\left(d_{1}+d_{2}+2\right)^{2}} & \text { if } x \in G_{1}, \\ \frac{d_{2}+2}{d_{1}+d_{2}+2} g_{\left(G_{2}, D_{2}\right)}(O, x)+\frac{\left(d_{1}+2\right)^{2} g_{1}-d_{1}\left(d_{2}+2\right) g_{2}}{\left(d_{1}+d_{2}+2\right)^{2}} & \text { if } x \in G_{2} .\end{cases}
$$

Then, we can easily check that $g$ is continuous on $G, \Delta(g)=\delta_{O}-\mu_{(G, D)}$ and $\int_{G} g \mu_{(G, D)}=0$. Thus, $g_{(G, D)}(O, x)=g(x)$. Therefore, by Lemma 4.1, we get the first formula. Moreover, using

$$
g_{(G, D)}(P, P)-2 g_{(G, D)}(O, P)+g_{(G, D)}(O, O)=r_{G}(O, P)=r_{G_{2}}(O, P)
$$

and

$$
g_{\left(G_{2}, D_{2}\right)}(P, P)-2 g_{\left(G_{2}, D_{2}\right)}(O, P)+g_{\left(G_{2}, D_{2}\right)}(O, O)=r_{G_{2}}(O, P),
$$

we obtain the second formula.

Corollary 4.3. Let $G$ be a connected metrized graph and $D$ an $\mathbb{R}$-divisor on $G$ with $\operatorname{deg}(D) \neq$ -2 . Let $C$ be a circle of length $l$. Then,

$$
e(G \vee C, D)=e(G, D)+\frac{\operatorname{deg}(D)}{3(\operatorname{deg} D+2)} l .
$$

Proof. Let $O=j(G \vee C)$ and $t: C \rightarrow[0, l)$ a coordinate of $C$ with $t(O)=0$. Then, it is easy to see that

$$
\mu_{(C, 0)}=\frac{d t}{l} \quad \text { and } \quad g_{(C, 0)}(O, x)=\frac{t(x)^{2}}{2 l}-\frac{t(x)}{2}+\frac{l}{12} .
$$

Thus, we have this formula by Proposition 4.2 .

Next, let's consider $e(G, D)$ for a segment.

Lemma 4.4. Let $G$ be a segment of length $l$, and $P$ and $Q$ terminal points of $G$. Let $a$ and $b$ be real numbers with $a+b \neq 0$, and $D$ an $\mathbb{R}$-divisor on $G$ given by $D=(2 a-1) P+(2 b-1) Q$. Then,

$$
e(G, D)=\left(\frac{4 a b}{a+b}-1\right) l, \quad g_{(G, D)}(P, P)=\frac{b^{2}}{(a+b)^{2}} l \quad \text { and } \quad g_{(G, D)}(Q, Q)=\frac{a^{2}}{(a+b)^{2}} l .
$$

Proof. First of all, by [12, Lemma 3.7],

$$
\mu_{(G, D)}=\frac{1}{a+b}\left(a \delta_{P}+b \delta_{Q}\right) .
$$

Let $t: G \rightarrow[0, l]$ be a coordinate of $G$. We set

$$
f(x)=-\frac{b}{a+b} t(x)+\frac{b^{2}}{(a+b)^{2}} l .
$$


Then, $\Delta(f)=\delta_{P}-\mu$ and $\int_{G} f \mu=0$. Thus, $f(x)=g_{(G, D)}(x, P)$. Therefore,

$$
g_{(G, D)}(P, P)=\frac{b^{2}}{(a+b)^{2}} l \quad \text { and } \quad g_{(G, D)}(P, Q)=g_{(G, D)}(Q, P)=-\frac{a b}{(a+b)^{2}} l .
$$

In the same way, we can see that

$$
g_{(G, D)}(Q, Q)=\frac{a^{2}}{(a+b)^{2}} l
$$

Therefore, we have

$$
e(G, D)=\left(\frac{4 a b}{a+b}-1\right) l
$$

This lemma can be generalized as follows.

Proposition 4.5. Let $G_{n}$ be a metrized graph given by the following figure.

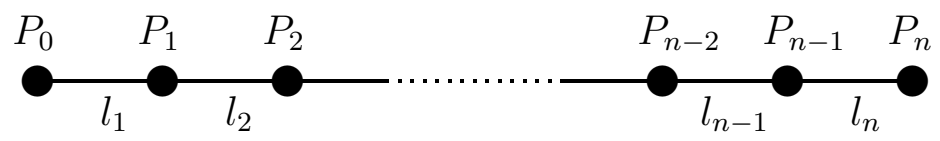

Let $l_{i}$ be the length between $P_{i-1}$ and $P_{i}$. Let

$$
D_{n}=\left(2 a_{0}-1\right) P_{0}+\left(2 a_{n}-1\right) P_{n}+\sum_{i=1}^{n-1} 2 a_{i} P_{i}
$$

be an $\mathbb{R}$-divisor on $G$ with $a_{i}>0$ for all $i$. Then, we have

$$
e\left(G_{n}, D_{n}\right)=\sum_{i=1}^{n}\left(\frac{4\left(a_{0}+\cdots+a_{i-1}\right)\left(a_{i}+\cdots+a_{n}\right)}{a_{0}+\cdots+a_{n}}-1\right) l_{i} .
$$

Proof. We set $e_{n}=e\left(G_{n}, D_{n}\right)$ and $t_{n}=g_{\left(G_{n}, D_{n}\right)}\left(P_{n}, P_{n}\right)$. We would like to prove

$$
e_{n}=\sum_{i=1}^{n}\left(\frac{4\left(a_{0}+\cdots+a_{i-1}\right)\left(a_{i}+\cdots+a_{n}\right)}{a_{0}+\cdots+a_{n}}-1\right) l_{i}
$$

and

$$
t_{n}=\frac{\sum_{i=1}^{n}\left(a_{0}+\cdots+a_{i-1}\right)^{2} l_{i}}{\left(a_{0}+\cdots+a_{n}\right)^{2}} .
$$

For this purpose, it is sufficient to show that

$$
t_{n+1}=\frac{\left(a_{0}+\cdots+a_{n}\right)^{2}}{\left(a_{0}+\cdots+a_{n}+a_{n+1}\right)^{2}}\left(t_{n}+l_{n+1}\right)
$$


and

$$
e_{n+1}=e_{n}+\frac{4 a_{n+1}\left(a_{0}+\cdots+a_{n}\right)}{a_{0}+\cdots+a_{n}+a_{n+1}} t_{n}+\left(\frac{4 a_{n+1}\left(a_{0}+\cdots+a_{n}\right)}{a_{0}+\cdots+a_{n}+a_{n+1}}-1\right) l_{n+1} \text {. }
$$

Let $L$ be a segment of length $l_{n+1}$, and $Q$ and $P$ terminal points of $L$. Let $E$ be an $\mathbb{R}$-divisor on $L$ given by $E=Q+\left(2 a_{n+1}-1\right) P$. Let's consider a one point sum $G_{n} \vee L$ obtained by joining $P_{n}$ and $Q$. Then, $G_{n+1}=G_{n} \vee L$ and $D_{n+1}=D_{n}+E$. Thus, by Proposition 4.2 and Lemma 4.4, we have the above recursive equations.

Corollary 4.6 (char $(k) \geq 0)$. Let $X$ be a smooth projective surface over $k, Y$ a smooth projective curve over $k$, and $f: X \rightarrow Y$ a generically smooth semistable curve over $Y$ of genus $g \geq 2$. Let $X_{y}$ be a singular fiber of $f$ over $y \in Y$. Let $X_{y}=C_{1}+\cdots+C_{n}$ be the irreducible decomposition of $X_{y}$. Let $G_{y}$ be the metrized graph given by the configuration of $X_{y}, v_{i}$ the vertex of $G_{y}$ corresponding to $C_{i}$, and $\omega_{y}$ the divisor on $G_{y}$ defined by $\omega_{y}=\sum_{i}\left(\omega_{X / Y} \cdot C_{i}\right) v_{i}$. If $X_{y}$ is a chain of stable components, then

$$
e_{y}=e\left(G_{y}, \omega_{y}\right)=\frac{g-1}{3 g} \delta_{0, y}+\sum_{i=1}^{\left[\frac{g}{2}\right]}\left(\frac{4 i(g-i)}{g}-1\right) \delta_{i, y}
$$

where $\delta_{i, y}$ is the number of nodes of type $i$ in $X_{y}$. In particular, if every singular fiber of $f$ is a chain of stable components, then

$$
\left(\omega_{X / Y} \cdot \omega_{X / Y}\right) \geq \frac{g-1}{3 g} \delta_{0}+\sum_{i=1}^{\left[\frac{g}{2}\right]}\left(\frac{4 i(g-i)}{g}-1\right) \delta_{i} .
$$

Moreover, if the above inequality is strict, then Bogomolov conjecture holds for the generic fiber of $f$.

Proof. Under our assumption, $G_{y}$ can be obtained by performing one sum of one segment and many circles. Thus, using Corollary 4.3 and Proposition 4.5, we can see the formula for $e_{y}$. For the last inequality, note that $\left(\omega_{X / Y}^{a} \cdot \omega_{X / Y}^{a}\right)_{a}=\left(\omega_{X / Y} \cdot \omega_{X / Y}\right)-\sum_{y} e_{y}$ and $\left(\omega_{X / Y}^{a} \cdot \omega_{X / Y}^{a}\right)_{a} \geq 0$ (cf. [12] or [8]). Furthermore, if $\left(\omega_{X / Y}^{a} \cdot \omega_{X / Y}^{a}\right)_{a}>0$, then Bogomolov conjecture holds for the generic fiber of $f$. (cf. [12])

\section{Bogomolov CONJeCtuRE OVER FUnCTION FIELDS}

Let $X$ be a smooth projective surface over $k, Y$ a smooth projective curve over $k$, and $f: X \rightarrow Y$ a generically smooth semistable curve of genus $g \geq 2$ over $Y$. Let $K$ be the function field of $Y, \bar{K}$ the algebraic closure of $K$, and $C$ the generic fiber of $f$. Let $j: C(\bar{K}) \rightarrow \operatorname{Jac}(C)(\bar{K})$ be a morphism given by $j(x)=(2 g-2) x-\omega_{C}$ and \|\|$_{N T}$ the semi-norm arising from the Neron-Tate height pairing on $\operatorname{Jac}(C)(\bar{K})$. We set

$$
B_{C}(P ; r)=\left\{x \in C(\bar{K}) \mid\|j(x)-P\|_{N T} \leq r\right\}
$$


for $P \in \operatorname{Jac}(C)(\bar{K})$ and $r \geq 0$, and

$$
r_{C}(P)= \begin{cases}-\infty & \text { if } \#\left(B_{C}(P ; 0)\right)=\infty, \\ \sup \left\{r \geq 0 \mid \#\left(B_{C}(P ; r)\right)<\infty\right\} & \text { otherwise. }\end{cases}
$$

An effective version of Bogomolov conjecture claims the following.

Conjecture 5.1 (Effective Bogomolov conjecture). If $f$ is non-isotrivial, then there is an effectively calculated positive number $r_{0}$ with

$$
\inf _{P \in \operatorname{Jac}(C)(\bar{K})} r_{C}(P) \geq r_{0} .
$$

In [8] and [9], we proved the following results.

(A) $(\operatorname{char}(k) \geq 0)$ If $f$ is non-isotrivial and the stable model of $f: X \rightarrow Y$ has only irreducible fibers, then

$$
\inf _{P \in \operatorname{Jac}(C)(\bar{K})} r_{C}(P) \geq \begin{cases}\sqrt{12(g-1)} & \text { if } f \text { is smooth, } \\ \sqrt{\frac{(g-1)^{3}}{3 g(2 g+1)} \delta_{0}} & \text { otherwise. }\end{cases}
$$

(B) $(\operatorname{char}(k) \geq 0)$ If $f$ is non-isotrivial and $g=2$, then $f$ is not smooth and

$$
\inf _{P \in \operatorname{Jac}(C)(\bar{K})} r_{C}(P) \geq \sqrt{\frac{2}{135} \delta_{0}+\frac{2}{5} \delta_{1}} .
$$

(According to the exact calculations in [9], $\left(\omega_{X / Y} \cdot \omega_{X / Y}\right)=\frac{1}{5} \delta_{0}+\frac{7}{5} \delta_{1}$ and $\sum_{y} e_{y} \leq$ $\frac{5}{27} \delta_{0}+\delta_{1}$.)

In this section, we would like to prove the following answer as an application of our slope inequality.

Theorem $5.2(\operatorname{char}(k)=0)$. We assume that $f$ is not smooth, every singular fiber of $f$ is a chain of stable components, and one of the following conditions:

(a) the generic fiber of $f$ is hyperelliptic, or

(b) every singular fiber of the stable model of $f: X \rightarrow Y$ has at most one node of type $i>0$.

Then we have

$$
\inf _{P \in \operatorname{Jac}(C)(\bar{K})} r_{C}(P) \geq \sqrt{\frac{(g-1)^{2}}{g(2 g+1)}\left(\frac{g-1}{3} \delta_{0}+\sum_{i=1}^{\left[\frac{g}{2}\right]} 4 i(g-i) \delta_{i}\right)} .
$$

Proof. First of all, note the following fact (cf. [12, Theorem 5.6], [8, orollary 2.3] or [9, Theorem 2.1]). If $\left(\omega_{X / Y}^{a} \cdot \omega_{X / Y}^{a}\right)_{a}>0$, then

$$
\inf _{P \in \operatorname{Jac}(C)(\bar{K})} r_{C}(P) \geq \sqrt{(g-1)\left(\omega_{X / Y}^{a} \cdot \omega_{X / Y}^{a}\right)_{a}},
$$


where $(\cdot)_{a}$ is the admissible pairing.

By the definition of admissible pairing, we can set

$$
\left(\omega_{X / Y}^{a} \cdot \omega_{X / Y}^{a}\right)_{a}=\left(\omega_{X / Y} \cdot \omega_{X / Y}\right)-\sum_{y \in Y} e_{y},
$$

where $e_{y}$ is $e\left(G_{y}, \omega_{y}\right)$ treated in $\S 4$. Under our assumption, by [2, Proposition 4.7] and Theorem 1.1, we have

$$
(8 g+4) \operatorname{deg}\left(f_{*}\left(\omega_{X / Y}\right)\right) \geq g \delta_{0}+\sum_{i=1}^{\left[\frac{g}{2}\right]} 4 i(g-i) \delta_{i} .
$$

Thus, using Noether formula, the above inequality implies

$$
\left(\omega_{X / Y} \cdot \omega_{X / Y}\right) \geq \frac{g-1}{2 g+1} \delta_{0}+\sum_{i=1}^{\left[\frac{g}{2}\right]}\left(\frac{12 i(g-i)}{2 g+1}-1\right) \delta_{i} .
$$

Moreover, by Corollary 4.6, we get

$$
\sum_{y} e_{y}=\frac{g-1}{3 g} \delta_{0}+\sum_{i=1}^{\left[\frac{g}{2}\right]}\left(\frac{4 i(g-i)}{g}-1\right) \delta_{i} .
$$

Thus, we have our theorem.

\section{REFERENCES}

[1] F. A. Bogomolov, Holomorphic tensors and vector bundles on projective varieties, Math. USSR-Izv., 13 (1978), 499-555

[2] M. Cornalba and J. Harris, Divisor classes associated to families of stable varieties, with application to the moduli space of curves, Ann. Scient. Ec. Norm. Sup., 21 (1988), 455-475.

[3] P. Deligne and D. Mumford, The irreducibility of the space of curves of given genus, Publ. Math. IHES, 36 (1969), 75-110.

[4] A. Paranjape and S. Ramanan, On the canonical ring of an algebraic curve, Algebraic Geometry and Commutative Algebra in Honor of Masayoshi NAGATA, vol. II (1987), 501-516.

[5] M. Maruyama, On a family of algebraic vector bundles, Number Theory, Algebraic Geometry and Commutative Algebra, in honor of Y. Akizuki, Kinokuniya, Tokyo, (1973), 95-146.

[6] A. Moriwaki, Arithmetic Bogomolov-Gieseker's inequality, Amer. J. Math. 117 (1995), 1325-1347.

[7] A. Moriwaki, Faltings modular height and self-intersection of dualizing sheaf, Math. Z., 220 (1995), $273-278$.

[8] A. Moriwaki, Bogomolov conjecture over function fields for stable curves with only irreducible fibers, to appear in Comp. Math..

[9] A. Moriwaki, Bogomolov conjecture for curves of genus 2 over function fields, preprint (1995), KyotoMath 95-13.

[10] D. Mumford, Stability of projective varieties, L'Ens. Math. 23 (1997), 39-110.

[11] G. Xiao, Fibered algebraic surfaces with low slope, Math. Ann., 276 (1987), 449-466.

[12] S. Zhang, Admissible pairing on a curve, Invent. Math., 112 (1993), 171-193.

Department of Mathematics, Faculty of Science, Kyoto University, Kyoto, 606-01, Japan 